\title{
Numerical analysis of freestream turbulence effects on the vortex-induced vibrations of a rectangular cylinder
}

\author{
Steven J. Daniels, Ian P. Castro, Zheng-Tong Xie* \\ Faculty of Engineering and the Environment,University of Southampton, SO17 1BJ, \\ Southampton, UK
}

\begin{abstract}
Large-Eddy Simulation of the flow around an elastically-mounted rectangular cylinder with an aspect ratio 4 was undertaken. 1DOF analysis of the heaving and torsional motions were performed under a free vibration. Various characteristics of the flow-field at lock-in are discussed. Subsequently, a divergence-free synthetic inflow generation approach was employed to analyse the effects of the freestream turbulence on the bridge response. The inflow turbulence intensity and integral length scales were systematically studied. The effect of turbulence intensity (up to $12 \%$ ) was shown to deplete the structural response for both torsional and heaving motions. A variation of the tested integral length scales, which were order of the cylinder dimensions, had less effects (than a variation of the turbulence intensity) on the structure response.
\end{abstract}

Keywords: large-eddy simulation; vortex-induced vibration; rectangular cylinder; turbulence effect.

*Email: Z.Xie@soton.ac.uk; Tel:+44 (0)23 80594493 
1

2

\section{Introduction}

Vortex Induced Vibrations (VIV) of a bluff body is an important fluidstructure interaction phenomenon, and many of questions concerning its mechanism remain unanswered. Recently, a review paper by Wu and Kareem (2012) describes a series of previous investigations on the VIV of bridge sections. A notable feature of this previous work is the enormous effort in determining the effects of the structure's geometry on the VIV response. For instance, a considerable amount of research concerns the circular cylinder, where the von Karman 'vortex street' is the main cause of the VIV.

However, cross-sections typical of a bridge deck have a number of sources for a VIV response due to the presence of an after-body and the inherent asymmetric feature. While much of the literature has focused on the amplitude incurred by VIV, comparatively few measurements are presented for the forces exerted on the body during lock-in. Therefore, further study would give a much deeper insight into the mechanism of VIV.

\subsection{Freestream turbulence effects on heaving responses}

Furthermore, literature concerning the VIV response under a turbulent flow is scarce. Usually, literature presents a bridge deck situated in a nominally smooth flow (typically with a turbulence intensity $<1 \%$ and not controlled/measured turbulence integral length scale), although bridges operate in the turbulent atmospheric boundary layer. Matsumoto et al. (1993) suggests that the effects of turbulence on VIV are rather complicated, this being mainly due to an interaction between the vortices in the wake (von Karman), and vortices induced by the structure's motion. According to Wu and Ka- 
reem (2012), the impacts of turbulence on the motion-induced forces are uncertain due to a limited understanding of this issue. Compared to the investigations of a static case, studies of the effect of free stream turbulence on flow-induced vibration of spring mounted cylinders are much scarcer. A very few experimental work on this are reported. Some of them are listed below.

Blackburn and Melbourne (1997) investigated the forced heaving vibration tests of a cylinder immersed in turbulent flow with analysis for the correlation and phase angles of the coefficient of lift. More recently, So et al. (2008) carried out a wind tunnel investigation, for the turbulent flow over a circular cylinder undergoing free vibration. They report a magnified response at lock-in under a turbulent flow, in comparison to smooth (or uniform) flow. Wu and Kareem (2012) speculated that freestream turbulence can stabilize or destabilize the response depending on the relative intensity of the von Karman to the motion-induced vortices; if the von Karman vortices dominate, the presence of freestream turbulence would reduce the structural response and vice-versa.

\subsection{Freestream turbulence effects on pitching responses}

It is also of great interest to study the pitching motion of the structure, and the torsional flutter responses. A notable contribution to torsional flutter is provided by Matsumoto (e.g. Matsumoto (2009)). Matsumoto has clarified the effects of von Karman vortices on torsional flutter, such as torsional mitigation (Matsumoto et al. (2003)). The effects of freestream turbulence on torsional response has rarely been addressed in the literature. Bartoli and Righi (2006) investigated the effects of turbulence on the torsional flutter in- 
stability, reporting that freestream turbulence has a stabilising effect on the response. This analysis is supported by calculations of the flutter derivatives, showing that the aerodynamic damping increased with the turbulence intensity of the freestream. It is suggested by Bartoli and Righi (2006) that the lack of correlation in the freestream turbulence reduced the correlation of pressure along the bridge span; this aspect however is not quantified in their work. Lin et al. (2005) carried out an investigation of a forced torsional oscillation tests of a cylinder of a bridge deck model. They subsequently report the effect of turbulence on the flutter derivatives, concluding that turbulence has a stabilising effect on flutter instability.

It is to be noted that the papers cited in the above paragraphs for freestream turbulence effects on VIV mainly consist of the experimental analysis. The experimental measurements provide ample amount of deflection data but fail to report the associated aerodynamic forces. It must be noted that a large portion of this topic is still not well understood yet, such as the characteristics of the aerodynamic forces at this occurrence, let alone the mechanism of von Karman vortices on the flutter stability.

1.3. Numerical analysis on the vortex-induced vibrations without and with considering freestream turbulence effects

Computational Fluid Dynamics (CFD) has become a powerful tool for the wind engineer. With the features associated with CFD, detailed analysis of heaving and pitching motion becomes more feasible, and will be very useful for further understanding of these topics. The use of CFD can provide a deeper insight into many fundamental topics, such as evaluating the effects of the geometrical features and freestream turbulence on the separated and 
reattaching flow past the sharp edges of the body (or bridge section).

Most of the literature concerning the analysis of VIV with CFD have largely been conducted in a two-dimensional domain. Fujiwara et al. (1993) applied such analysis to the bridge deck of a $\mathrm{H}$ cross-section. Using the Arbitrary Eulerian Equations (ALE), their analysis showed that there is a sudden change in lift and amplitude of the section at two distinct Reynolds numbers (i.e. 1000, 2400). A notable contribution is the work of Lee et al. (1997). In this work the cross-sections of the Namehae and Seohae bridges were analysed using URANS turbulence modelling while subjecting the models to a forced vibration. Their results show a good agreement of the aerodynamic forces with the equivalent wind tunnel tests for the Namehae bridge, and with the test of structural response amplitudes for the Seohae bridge.

By using the Unsteady-Reynolds Averaged Navier-Stokes (URANS) approach, Fransos and Bruno (2010) investigated the characteristics of the shear layers around a fixed trapezoidal-shaped bridge section with varying corner degree-of-sharpness and turbulent length scale (with low turbulence intensity). The authors note the sensitivity of the shear layer separation around the bridge section with Reynolds number and turbulent length scale. From this they identified various regimes for the local and global flowfield and the effects on the aerodynamic forces. In a later work, Bruno and Fransos (2011) analysed the same features over the bridge section using a probabilistic approach.

Sarwar et al. (2008) investigated the rectangular and box-girder crosssection, with and without fairings using Large Eddy Simulation (LES). Their work applied a forced vibration to the structure, focusing on the phase-angle 
changes, and lift force characteristics around the lock-in region. Their later work (Sarwar and Ishihara (2010)) also presents the structural response for the free oscillations, though mainly focuses on the flow-field and aerodynamic characteristics for the forced motion.

In many cases of analysis, the data from CFD have been complementary to the equivalent wind tunnel study. More recently this aspect has been reciprocated. Marra et al. (2015) carried out a systematic wind tunnel study of the VIV response of an elongated rectangular cylinder $(B / D=4)$ with various Scruton numbers. Their work provides benchmark data for different models and CFD techniques, as well as suggesting a new model for predicting the amplitude of the cylinder at lock-in with different Scruton numbers.

\section{4. outline of the paper}

Literature on numerically modelling the torsional responses of the bridge deck are extremely scarce. To the best of the authors' knowledge, analysis using a numerical approach considering freestream turbulence effects is not reported in the literature.

In this paper, using LES we examine the flow around a rectangular cylinder (assimilating a simplified bridge deck) under smooth and turbulent flows while considering the underlying mechanisms affecting the VIV response. The chosen side ratio for the cylinder was 4, representing an extreme case of a bridge section. At this ratio, the effects of galloping on the VIV response is suggested to be minimal (Mannini et al. (2014)). For a side ratio greater than 3 , the vortex shedding in the wake is triggered by the impinging shear layers from the leading edge of the cylinder. The impinging shear layers exhibit different modes of vortex shedding depending on the side ratio. However, 
for the present work, only the first mode of vortex shedding is considered to produce the VIV response.

The objective of this paper is to further understand and quantify the effects of freestream turbulence (in terms of intensity and integral length) on the vortex-induced vibrations of a simplified structure. $\S 2$ shows the setup conditions for LES which are adopted from appropriate wind tunnel tests. $§ 3$ briefs the computational models, including LES for the turbulence flows and the structure model, etc. $§ 4$ presents baseline studies - modelling vortex-induced vibrations of a rectangular cylinder in smooth flows, including heaving and pitching response. $§ 5$ studies freestream turbulence effects on the heaving and pitching response. $\S 6$ presents further data analysis, i.e. on spanwise correlation of pressure fluctuations on the cylinder surface.

\section{Adopted setup conditions}

\subsection{Setup conditions for heaving response}

For comparison of the fluid-structure coupling method for the heaving motion, the settings of the numerical model were in accordance with those of the wind tunnel of Marra et al. (2011). The model is a rectangular bridge deck with the height of the cross section (D) as $0.075 \mathrm{~m}$, width (B) $0.3 \mathrm{~m}$, and length (spanwise) 1m. A uniform smooth flow $\left(I_{u}<0.1 \%\right)$ was specified with the Reynolds number 40,000 (based on freestream velocity $\mathrm{U}$ at lock-in and the cylinder thickness D).

An initial displacement of $0.1 D$ was imposed so the vibration could converge quickly to the VIV response. The effective structural damping is known to increase with the amplitude of response. For VIVs being a self-limiting 
process, this aspect is not considered in this investigation. Hence, the Scruton number $S c$ is assumed to be constant throughout the lock-in region. This number, based on the logarithmic decrement $\delta$ or structural damping $\zeta$ is defined as

$$
S c=\frac{2 m \delta}{\rho_{f} D^{2}}=\frac{4 \pi m \zeta}{\rho_{f} D^{2}} .
$$

The structural damping was deduced by the relation $\delta=2 \pi \zeta$. An important consideration is the choice of a suitable Scruton number to accurately reproduce the structural response under a free oscillation. To be consistent with the wind tunnel experiment of Marra et al. (2011), the structural parameters were chosen with the Scruton number $S c=6$. The corresponding mass per unit length $m$ was $6.085 \mathrm{Kg} / \mathrm{m}$, and structural damping $\zeta$ was $0.21 \%$. The natural frequency of the structure $f_{n}$ was set as $13.43 \mathrm{~Hz} . \rho_{f}$ is the air density.

\subsection{Setup conditions for pitching response}

The wind tunnel results by Matsumoto et al. (2008) were used for comparison with the simulations for pitching response. The experimental parameters in Matsumoto et al. (2008) were adopted in the simulations. The model section is the same as that in $\S 2.1$, i.e. a rectangular bridge deck with the height of the cross section (D) as $0.075 \mathrm{~m}$ and the width (B) $0.3 \mathrm{~m}$. A uniform smooth flow was specified with the Reynolds number 40,000 (based on freestream velocity $U$ at lock-in and the cylinder thickness $D$ ), which is close to the Reynolds number in Matsumoto et al. (2008). Given the effect of the Reynolds number in this range is weak for flows around such a bluff body with sharp edges, we don't expect evident discrepancy due to a small difference of Reynolds number. 
Again, the structural damping is assumed to be constant with the Scruton number $S c=7.862$ under the definition:

$$
S c=\frac{2 I \delta}{\rho_{f} D^{4}}=\frac{4 \pi I \zeta}{\rho_{f} D^{4}} .
$$

The corresponding mass moment of inertia per unit length $I$ was $0.01494 \mathrm{Kg} m$, and structural damping $\zeta$ was $0.162 \%$. The natural frequency of the structure $f_{t}$ was set as $21.5 \mathrm{~Hz}$.

\subsection{Freestream turbulence conditions}

For the numerical simulations of the heaving and pitching motion in freestream turbulence effects, the streamwise turbulence intensity $\left(I_{1}=u^{\prime} / U\right)$ was set $6 \%$ as the 'base setting', following the observations of Matsumoto et al. (1993). It was reported in Castro et al. (2006) and Xie and Castro (2008) that the turbulence integral length scales of flows over an array of bluff bodies are of the same order of magnitude of the characteristic length of the bluff body. In this study, the integral length scales $L_{11}$ (streamwise), $L_{22}$ (vertical) and $L_{33}$ (spanwise) of the 'base setting' are respectively $2 B / 3$, $2 B / 9$ and $B / 3$, where $B$ is the bridge width.

\section{Computational modelling}

The calculations in this work were performed using the open-source code OpenFOAM. The used models in OpenFOAM previously have been tested for simulating plane channel flows (Kim et al. (2013)), bluff body flows (Daniels et al. (2013)), and wind turbine blade flows (Kim and Xie (2016)). 


\subsection{Turbulence model}

The high fidelity turbulence model - Large-Eddy Simulation (LES) was performed throughout this work. The filtered continuity and Navier-Stokes equations of LES are written as follows,

$$
\begin{aligned}
& \frac{\partial u_{i}}{\partial x_{i}}=0 \\
& \frac{\partial u_{i}}{\partial t}+\frac{\partial u_{i} u_{j}}{\partial x_{j}}=-\frac{1}{\rho}\left(\frac{\partial p}{\partial x_{i}}\right)+\frac{\partial}{\partial x_{j}}\left(\tau_{i j}+\nu \frac{\partial u_{i}}{\partial x_{j}}\right) .
\end{aligned}
$$

The dynamical quantities, $u_{i}, p$ are resolved-scale (filtered) velocity and pressure respectively, and $\tau_{i j}$ is the subgrid-scale (SGS) Reynolds stress. The Mixed Time Scale (MTS) model proposed by Inagaki et al. (2005) is used to model the SGS Reynolds stress term. This SGS model has the feature of requiring no wall damping function. The constants associated with the MTS model, $C_{M}$ and $C_{T}$, were specified as 0.05 and 10 respectively. These are in accordance with Inagaki et al. (2005), who optimised these values for bluff body flows. This model has also been used in Kim and Xie (2016).

A no-slip boundary condition was applied to the surfaces of the square cylinder. For the outflow, a zero-gradient (von Neumann) boundary condition was imposed. The symmetry boundary condition was prescribed for the top and bottom boundaries, while periodic conditions were imposed to the lateral sides of the domain. For the smooth flow cases $(\S 4)$, a Dirichlet condition for the velocity field was applied to the inlet boundary.

\subsection{Inflow turbulence generation for $L E S$}

The approach in Xie and Castro (2008), which is denoted Hybrid Forward Stepwise (HFS) method, imposes correlations using an exponential function 
to satisfy the prescribed space and time integral length scales. It is a synthetic turbulence generation method. The inlet velocities can be written as,

$$
u_{i}=U_{i}+a_{i j} u_{*, j}
$$

where $i, j=1,2,3 . u_{i}$ is an instantaneous velocity which is imposed at the inlet boundary, $U_{i}$ is a prescribed mean velocity, $a_{i j}$ is a prescribed tensor (Eq.5) and $u_{*, j}$ is an auto-correlated fluctuation satisfying the prescribed integral length scales, but with a zero mean, zero cross-correlations and a unit variance. Lund et al. (1998) suggested a form for $a_{i j}$, using Cholesky decomposition of the prescribed Reynolds stress tensor, $R_{i j}$,

$$
a_{i j}=\left(\begin{array}{ccc}
\sqrt{R_{11}} & 0 & 0 \\
R_{21} / a_{11} & \sqrt{R_{22}-a_{21}^{2}} & 0 \\
R_{31} / a_{11} & \left(R_{32}-a_{21} a_{31}\right) / a_{22} & \sqrt{R_{33}-a_{31}^{2}-a_{32}^{2}}
\end{array}\right) .
$$

This matrix builds scaling and cross-correlations based on $u_{*, j}$ in Eq. 4 . To impose correlations on random sequences, the HFS approach adopts an exponential function instead of a Gaussian function used in the early digitalfilter based methods. The digital filter method was used to generate spatial correlations,

$$
\psi_{m}=\sum_{j=-N}^{N} b_{j} r_{m+j},
$$

where $N=2 n, n=L / \Delta x, \Delta x$ is grid size and $L$ is integral length scale. $\psi_{m}$ is the intermediate velocity field and $r_{j}$ is a one-dimensional random number sequence with a zero mean and a unit variance. $\psi_{m}$ is a one-dimensional number sequence with a zero mean, a unit variance and spatial correlations. 
Note that the subscripts, $m, j$, are the position indices. The constant $b_{j}$ is estimated as,

$$
b_{j}=\frac{b_{j}^{\prime}}{\left(\sum_{l=-N}^{N} b_{l}^{\prime 2}\right)^{1 / 2}} \text { with } b_{j}^{\prime}=\exp \left(-\frac{\pi|j|}{2 n}\right) .
$$

It is straightforward to generate spatial correlations for a two dimensional space (cf. Eq.6) as,

$$
\psi_{m, l}=\sum_{j=-N}^{N} \sum_{k=-N}^{N} b_{j} b_{k} r_{m+j, l+k} .
$$

It is to be noted that only one slice of two dimensional data, $\psi_{m, l}$, is generated at each time step. Based on these data, a time correlation is built using the efficient forward stepwise relation,

$$
u_{*, i}(t+\Delta t)=u_{*, i}(t) \exp \left(-\frac{C_{X C} \Delta t}{T}\right)+\psi_{i}(t)\left[1-\exp \left(-\frac{2 C_{X C} \Delta t}{T}\right)\right]^{0.5}
$$

where the constant $C_{X C}=\pi / 4$ and $T$ is the Lagrangian time scale which is estimated using $T=L / U_{1}$ where, again, $L$ is a turbulence integral length scale and $U_{1}$ is a mean convective velocity. Note that in Eq.9 the subscript $i$ is a vector index, i.e. $i=1,2,3$. The HFS method generates synthetic turbulence by using Eqs. 4 - 9. By using exponential correlations, in particular in the streamwise direction, it significantly reduces the computational time compared to the early digital filter based approaches. The HFS method is a combination of the digital filter method and the Forward Stepwise Method (Kim et al. (2011)). 
Based on the HFS method, Kim et al. (2013) develop a divergence-free approach - denoted DHFS thereafter. After the predictor step in the PISO solver for unsteady flows, synthetic turbulence fluctuations are inserted into the source term of the Poisson equation in one of the corrector steps. Hence the divergence-free condition was achieved without solving an additional Poisson equation. The DHFS approach significantly improve the prediction of surface pressure fluctuations. More details of the implementation of the DHFS approach is given in the following sub-section. The DHFS has been tested in Daniels et al. (2013), and Kim and Xie (2016).

For the freestream turbulence cases $(\S 5)$, the DHFS is used to generate the inflow turbulence. In order to satisfy the divergence-free criterion during pressure-velocity coupling, DHFS imposes the synthetic turbulence downstream from the inlet boundary at a distance $x_{0}$. For the present work, $x_{0}=B / 2$. The turbulence generation approach requires a set of integral length scales, and turbulence intensity. Again, the streamwise turbulence intensity $\left(I_{1}=u^{\prime} / U\right)$ was specified as $6 \%$. The integral length scales $L_{i j}$ were defined as

$$
L_{i j}=\int_{0}^{r_{i j, 0.1}} C_{i}\left(r \hat{e}_{j}\right) d r,
$$

where $C_{i}\left(r \hat{e}_{j}\right)$ is the correlation function. The indices $\mathrm{i}$ and $\mathrm{j}$ indicate the velocity vector and directions respectively. $r_{i j, 0.1}$ is the separation distance for function, which is set equal to 0.1 . The integral length scale $L_{11}$ was chosen to be $2 B / 3$; the components of pairs $\left(I_{2}=v^{\prime} / U, L_{22}\right)$ and $\left(I_{3}=w^{\prime} / U, L_{33}\right)$ were taken as $1 / 3$ and $1 / 2$ respectively of the corresponding component of the pair $\left(I_{1}, L_{11}\right)$. These turbulence parameters are denoted as the 'base 
$I_{1}, I_{2}, I_{3}$ ' and 'base $L_{11}, L_{22}, L_{33}$ ' respectively for the turbulence intensities and length scales. The calculations were run with the same initialising and averaging time as the smooth flow cases.

\subsection{Structure model}

As this work focuses on the free vibration of the cylinder, a two-way coupling is required between the fluid and the structure. For an efficient calculation, a partitioned procedure was chosen. The fluid-structure algorithm was similar to that of the Conventional Sequential Staggered (CSS) procedure. A similar approach has been implemented in Sarwar and Ishihara (2010), and Placzek et al. (2008) for a forced oscillation using an Ordinary Differential Equation (ODE) to prescribe the motion of the cylinder. In the present work, the response of the structure was calculated using a forced mass-spring-damper equation.

For heaving motion (in $\S 4.1$ ), the governing equation of the structure is written as follows,

$$
m\left(\ddot{y}+2 \zeta \omega_{n} \dot{y}+\omega_{n}^{2} y\right)=\frac{1}{2} C_{L}(t) \rho_{f} U^{2} B
$$

where $m$ is the mass per-unit-length of the structure, $\zeta$ is the damping ratio, $\omega_{n}$ is the circular natural frequency of the structure in the vertical direction, $\rho_{f}$ is the fluid density, $\mathrm{U}$ is the freestream velocity, and $\mathrm{B}$ is the streamwise length of the cylinder. The time-dependent lift coefficient $C_{L}(t)$ was obtained by integrating the pressure over the surface of the cylinder. Eq. 11 is integrated for each time step using the Runge-Kutta-Fehlberg method.

For the pitching motion (in $\S 4.2$ ), the governing equation of the structure is written as follows, 


$$
I\left(\ddot{\theta}+2 \zeta \omega_{t} \dot{\theta}+\omega_{t}^{2} \theta\right)=\frac{1}{2} C_{m}(t) \rho_{f} U^{2} B^{2} .
$$

where $I$ is mass moment of inertia per-unit-length of the structure, $\zeta$ is the damping ratio, $\omega_{t}$ is the circular natural frequency of the structure of pitching, $C_{m}(t)$ is the pitching moment.

\subsection{Dynamic mesh}

The calculated deflection of the square cylinder was used for the dynamic mesh. The term dynamic mesh refers to the relative distances among grid points changing in time to adjust to an unsteady motion of a body. This can be achieved through squeezing and stretching the surrounding cells and their associated vertices. For the finite volume method, the conservation equation of property, $\phi$, over an arbitrary moving control volume, $V_{C}$, in integral form is

$$
\frac{d}{d t} \int_{V_{C}} \phi d V_{C}+\int_{A} d A \cdot\left(\vec{u}-\vec{u}_{b}\right) \phi=\int_{V_{C}} \nabla \cdot(\Gamma \nabla \phi) d V_{C},
$$

where $\vec{u}$ is the fluid velocity vector, $A$ is the cell-surface-normal vector and $\overrightarrow{u_{b}}$ is the boundary velocity vector of the cell-face. To govern the vertex motion, OpenFOAM adopts a Laplacian smoothing scheme, described by

$$
\nabla \cdot\left(\gamma \nabla u_{p}\right)=0
$$

where $u_{p}$ is the point velocity, which is imposed at each vertex of the control volume. The boundary velocity $u_{b}$ is interpolated from $u_{p}$. The boundary conditions for equation 14 are enforced from the known boundary motion, 
e.g. a moving wall. The vertex position at the time level $n+1$ is calculated by using $u_{p}$,

$$
x^{n+1}=x^{n}+u_{p} \Delta t .
$$

The variable $\gamma$ prescribes the distribution of deforming cells around the moving body. Ideally for the Laplacian approach, the cell distortion near the moving wall should be less perturbed by the motion of the body, while with increasing distance away from the wall, the cells should have a greater freedom to deform. Under this concept, the quadratic diffusion model $(\gamma=$ $\left.1 / l^{2}\right)$ has shown to present a suitable distribution of cells around the body (Jasak and Tuković (2004)), with $l$ being the distance from the moving wall. Hence, this model is adopted for the present work. As the grid motion in the whole domain is governed by equation 14 , an interface between the static and dynamic mesh regions is not required.

\subsection{Numerical approach}

A second order implicit scheme was used for the temporal discretisation and the bounded Gamma scheme (Jasak (1996)) was used for the convection term. For the latter, the chosen value of $\beta$ determines the blending between Central differencing and Upwind differencing. In this work, $\beta$ was set as 0.1, as suggested by Jasak (1996). The PIMPLE algorithm was adopted for the velocity-pressure coupling, combining the SIMPLE and PISO (Issa (1985)) algorithms. The momentum equation are solved repeatedly as outer iterations (SIMPLE), while pressure corrections are performed using the PISO algorithm. The number of outer corrections was set to 2 , and the number of pressure correctors was set to 3 in this study. 
The dimensions of the computational domain were $66.6 D \times 20 D \times 13.3 D$, with a rectangular cylinder placed at $24 \mathrm{D}$ downstream from the inlet; D is the cylinder's thickness. A block-structured mesh was constructed. The $y_{1}^{+}$of the cells around the surface of the cylinder was set to be within the range $y_{1}^{+}<5$ (i.e. equivalent to $D / 200$ ) with a growth rate of 1.05 . The parameter, $\delta z / D$, has widely been used for cylinder flows, with $\delta z$ being the grid size in the spanwise direction. Bruno et al. (2012) varied this parameter between 0.05 to 0.21 , while plotting the spanwise correlation. Their results show that a value of 0.21 produced a larger correlation of pressure around the leading edge, when compared to the equivalent experimental result. Bruno et al. (2012) also found that the spanwise correlation for the $\delta z / D=0.1$ and 0.05 resolution showed little difference to the result. As modelling this region is crucial for the VIV response (e.g. Shiraishi and Matsumoto (1983), Matsumoto et al. (2008)), it is important to resolve the flow sufficiently. Therefore, also considering obtaining an efficient calculation, the resolution $\delta z / D=0.1$ was adopted for the present work. This is also consistent with the minimum requirement $(\delta z / D \leq 0.1)$ specified by Tamura et al. (2008). The overall distribution of the mesh within the 3D domain is shown in Fig. 1 , with the origin of the reference system placed at the left bottom corner of the inlet plane when looking downstream.

The time duration for initialising the calculation of all cases was set to 200, 000 time steps with $\Delta t=10^{-5}$ secs. This step size was chosen in order to keep the CFL number $(U \Delta t / \Delta x)$ less than 1 ( $\Delta x$ is the smallest grid size in the computational domain). This is equivalent to $220 t^{*}$, where $t^{*}=U t / D$, and was adequate to achieve the VIV response. 
In order to optimise the sampling duration for the calculation of spanwise correlation of surface pressure, the convergence of the correlation coefficient was checked as in Bruno et al. (2010). The convergence of the chosen parameter is assessed for increasing extents of a non-dimensional time window $T_{k}^{*}$ within the sampled time series, where $T_{1}^{*}=50, T_{k}^{*}=T_{k-1}^{*}+50$. The percentage residual for the chosen parameter, $\phi$, is evaluated at the at the $k^{\text {th }}$ sampling window as $\phi_{\text {res }}=100\left|\left(\phi_{k}-\phi_{k-1}\right)\right| / \phi_{k}$. A sampling duration $T_{s}^{*} \geq 400$ has been found to be required to have a residual less than $5 \%$. This duration corresponds to approximately 47 periods of the oscillating cylinder in the lock-in region $\left(U_{r}=U / f_{n} D=8.4\right)$. The same process was used for all cases.

\section{Smooth flow response}

\section{1. heaving response}

Response amplitudes of the numerical method compared with wind tunnel data (Marra et al. (2011)) are presented in Fig. 2. Marra et al. (2011) repeated their experiment twice with small differences between the two. The first set of results (labelled 'series 1' in their paper) is presented for comparison. It can be seen that the numerical approach has adequately determined the statistics of the deflection over the lock-in region. We calculated that the Strouhal number $\left(f_{s} D / U\right.$, where $f_{s}$ is the shedding frequency) for a static case was 0.134, while Marra et al. (2011) estimated this to be 0.136. Despite this small discrepancy, the present value is within the range of Strouhal numbers for rectangular cylinders, according to Shimada and Ishihara (2002). Phase-averaged streamlines around the cylinder, and the corresponding 
pressure distribution over the lateral surfaces during one cycle of the structural response at lock-in $\left(T_{n}=1 / f_{n}\right)$ are presented in Fig. 3. It has been noted in the literature (e.g. Sarpkaya (2004)) that the relative peak amplitude at lock-in is not constant and the motion is not purely sinusoidal, and is largely determined by the conditions of the previous cycle. Consequently, the instantaneous states of self-excited vibrations at the same amplitude and average frequency do not necessarily result in the same pressure distribution and flow field. Therefore, the data presented in Fig. 3 were obtained by phase-averaging over 10 cycles and also spanwise averaging with a satisfactory convergence. These data correspond to the lock-in response at reduced velocity $U_{r}=8.4$.

The time-series graph on the top of Fig. 3 shows the phase-averaged deflection and lift progression over one cycle. It can be seen that the phase lag between the structural motion and the lift is approximately $90^{\circ}$ (i.e. $T_{n} / 4$ ). It should be noted that this phase lag does fluctuate between $80-120^{\circ}$ in the raw data.

Fig. 3(i-iv) respectively correspond to phase angles $0^{\circ}, 90^{\circ}, 180^{\circ}$ and $270^{\circ}$ during one cycle, which are indicated on the time-series graph. The vortices formed at the leading edge in these figures are denoted two-characters markers "**". The first character 'A' or 'B' are for the top and bottom surfaces respectively. The 2 nd character is a 1-digit number indicating the order of the vortex. The progression of the flow around the cylinder is as follows:

Fig. 3(i): The cycle begins at the rest position $(y=0)$ at phase $0^{\circ}$. The corresponding streamline diagram shows that a leading edge vortex (de- 
noted 'A1') is formed on the top surface of the section; a peak (positive) lift is attained by this vortex formation. Meanwhile, on the bottom surface, the vortex created from the previous half-cycle ('B1') convects along the cylinder at approximately $50 \%$ of the freestream velocity. This observation is consistent with the suggestion of Matsumoto et al. (2008), who speculated that the leading edge vortices must convect along the cylinder at this velocity in order for a peak response to occur between reduced velocities $3-4$.

Fig. 3(ii): A positive peak deflection (i.e. $y / D=0.05$ ) is reached at phase $90^{\circ}$. The vortex formed on the top surface has grown since (i), causing a concentration of pressure (approximately $C_{p}=-2$ ) towards the leading edge of the cylinder. 'B1' continues to convect along the bridge section at the same velocity as in (i). At the same time, a second vortex 'B2' is formed on the bottom surface. The combined effect of A1, B1 and B2 results in a nearly zero lift.

Fig. 3(iii): The cylinder returns to the original rest position (i.e. $y=0$ ) at phase $180^{\circ}$. It can be seen that the flow field is a mirrored one to (i) about the horizontal centreplane of the cylinder, with a small difference in vortex formation towards the leeward corner. This difference however does not seem to have a significant effect on the pressure distribution in this region. Fig. 3(iv): A negative peak deflection (i.e. $y / D=-0.05$ ) is reached at phase $270^{\circ}$. Again, it can be seen that the flow field is a mirrored one to (ii) about the horizontal centreplane of the cylinder. For the transition from (iv) to (i) in the next cycle, 'A2' becomes 'A1' and 'B2' becomes 'B1'.

Such a relation between the lift and the structural motion is repeated at each 
cycle. Nevertheless, the simulated sequence presented here is consistent with the explanation of the pressure formation over the cylinder described by Komatsu and Kobayashi (1980), based on their experimental investigation. It is to be noted that this surface pressure in Fig. 3 demonstrates the importance of the leading edge vortices during lock-in. The significance of this is further discussed in the proceeding sections.

One characteristic of the heaving response of the structure is the formation of the leading edge vortices, which are more prominent than the equivalent static case. Hence, these leading edge vortices are commonly referred to as Motion-Induced-Vortices (MIVs). This identification has been popularised by a few reports (e.g. Komatsu and Kobayashi (1980); Matsumoto (1996); Matsumoto et al. (2008)). It is also shown in $\S 4.1$ that the MIVs convect along the lateral sides and eventually interact with von Karman wake vortices. This interaction is rather complicated, especially after lock-in.

\subsection{Pitching response}

In the pitching motion, the MIVs play an even more important role in the bridge response.

The r.m.s pitching angle versus reduced-velocity diagram of the torsional motion is shown in Fig. 4. The rotational axis is the mid-chord of the cylinder. The numerical results show a close agreement with the equivalent wind tunnel data (Matsumoto et al. (2008)) for the first peak located approximately at the reduced velocity 5.1. The location of this self-limiting peak is in agreement with the guidelines by Shiraishi and Matsumoto (1983), which proposes the locations of a VIV response to be roughly two-thirds of the inverse Strouhal number. There is some discrepancy however for the onset 
of the lock-in region, which might be due to the differences in Strouhal number described for the heaving case. The pitching response begins to diverge from reduced velocity 10 . The onset reduced velocity of this is approximately twice that of the first peak response. This is consistent with that in Kawatani et al. (1999) for a rectangular cylinder with the same aspect ratio $B / D=4$. To the best of the authors' knowledge, validation data of the pitching angles beyond reduced velocity 10 is not present in the literature for this particular case.

Similar to the heaving motion cases in $\S 4.1$, streamlines around the bridge section, and the corresponding pressure distribution over the lateral surfaces during one cycle $\left(T_{n}=1 / f_{t}\right)$ of the structural response at a reduced velocity 4.9 are presented in Fig. 5. These data were processed in a similar way to those in $\S 4.1$. Fig. 5 is also presented in a similar way as Fig. 3. A distinct characteristic of the phase averaged moment coefficient $\left(C_{M}=M / 0.5 \rho_{f} U^{2} B^{2}\right)$ is the presence of a double peak when the pitching motion is approaching its maximum. Each peak corresponds the formation of a leading edge vortex. The first vortex is formed at the rest position $(y=0)$ (e.g. Fig. 5(i) vortex A1) and the second is formed at the maximum amplitude (e.g. Fig. 5(ii) vortex B3). Both vortices coalesce at the centre $(B / 2)$ and convect into the wake, then interact with a von Karman vortex formed on the opposing side resulting in a pair of vortices - denoted 'P' vortices in the literature (e.g. Williamson and Govardhan (2004)). The pressure distributions of the pitching motion during a cycle is similar to that of the heaving motion, but with a greater peak, and a narrower distribution at the leading edge formed at the peak deflections. 
Numerical simulations of the same cross section were attempted by Shimada and Ishihara (2012), who used an unsteady two-dimensional k- $\epsilon$ model for reduced velocities 12 and 25 at the divergence region. The choice of turbulence model gives a somewhat limited insight into the dynamics of the leading edge vortex, presenting only a single large vortex formed at the peak amplitudes, whereas the current LES modelling is able to provide a deeper insight into the vortex formation and the resulting peak loading during each cycle.

\section{Freestream turbulence effects on the heaving and pitching re- sponse}

To check the effectiveness at the inflow generation, Fig.6 shows a comparison power spectral density of velocity fluctuations at $x=3 B, \mathrm{y}=2.5 \mathrm{~B}$ on the central plane, with the von Karman wind spectra (ESDU-85020, 2001). The three velocity components for the von Karman spectra are described,

$$
\begin{gathered}
\frac{P S D\left(u^{\prime}\right)}{\sigma_{u}^{2}}=\frac{4 n_{u}}{f\left(1+70.8 n_{u}^{2}\right)^{5 / 6}} ; n_{u}=f L_{u} / U_{\text {avg }} \\
\frac{P S D\left(\xi^{\prime}\right)}{\sigma_{\xi}^{2}}=\frac{4 n_{\xi}\left(1+755.2 n_{\xi}^{2}\right)}{f\left(1+283.2 n_{\xi}^{2}\right)^{11 / 6}} ; n_{\xi}=f L_{\xi} / U_{\text {avg }} ; \xi=v \text { or } w
\end{gathered}
$$

where $L$ is the integral length scale, $f$ is the frequency, $\sigma^{2}$ is the variance, and $U_{\text {avg }}$ is the local average velocity. The LES spectra show an evident $-5 / 3$ slope. However, at very high frequencies the LES spectra show a slightly steeper slope, which is due to the limited resolution in this region. 
It is to be noted that besides the adequate turbulence generation, a sufficient spatial resolution downstream of the inlet has also be designed (Fig. 1) to ensure adequate turbulent content approaching the cylinder. We have carefully checked the loss of Turbulent Kinetic Energy (TKE) from $x / B=1$ to 3.33 at the cylinder height, and have found it is less than $10 \%$. From $x / B$ $=2.67$ to 3.33 , the TKE is almost constant. Since the resolution downstream from $x / B=3.33$ until the cylinder is finer than upstream, the TKE loss in this region is estimated very small.

The responses for the heaving motion around lock-in under incoming turbulent flows are presented in Figs. 7 and 8. Fig.7 presents the response of the cylinder with the base settings for turbulence intensity with varied integral length scales. The integral length scales $L_{11}, L_{22}, L_{33}$ were equal to, or double or half of the corresponding component of the base settings. The ratios between the length scales $\left(L_{11}, L_{22}, L_{33}\right)$ were maintained the same. Fig. 7 shows that in general the structure response increases with the increase of the turbulence length scale. Nevertheless, variance of the integral length scale within the tested range has only a moderate influence on the structural response.

The effects of turbulence length scale on the surface pressure fluctuations on a blunt plate $(B / D \geq 4)$ was previously studied by Li and Melbourne (1999). In their study with a constant turbulence intensity $I_{1}=8 \%$ and various length scales $L_{11}$, the peak pressure increases with the increase of length scales $L_{11}$. The present study confirm a similar trend. To further confirm this observation, Fig.9(a) shows the ratio of peak response $\left(R_{A}=y_{\text {turb. }} / y_{\text {smooth }}\right)$ between the smooth and turbulent flows versus $L_{11} / B$ at reduced velocity 
$U / f_{n} D=8.4$.

The effects of turbulence intensity on the structural response were also studied, and are presented in Fig.8. Matsumoto et al. (1993) report that the response under the 'base settings' is approximately half of the response in a smooth flow. The present results agree well with this observation. To further understand the effects of the freestream turbulence intensity, the turbulent intensities $\left(I_{1}, I_{2}, I_{3}\right)$ were subsequently doubled and halved. The ratio between the three components were maintained the same. Clearly the response decreases monotonously with the increase of the turbulence intensity.

Again the ratio of peak response $\left(R_{A}=y_{\text {turb }} / y_{\text {smooth }}\right)$ between the smooth and turbulent flows versus the streamwise component $I_{1}$ is presented in Fig. 9(b). These results seem to present a counter-intuitive relation between the structural response and turbulence intensity. Our previous work (Daniels et al. (2013)), and other literature (e.g. Li and Melbourne (1995)) demonstrate that the freestream turbulence intensity generally enhances the r.m.s surface pressure fluctuations on a bluff body, which "by extension" should enhances the structural response. Clearly, some more crucial mechanism influences the VIV. Considering our work for the turbulence length scales, it can be deduced that there is some correlation between the eddies of the freestream turbulence, and MIV and subsequently the structural response. More specifically, the turbulence with the integral length scales in the current tested range must reduce the strength of the MIV formed at the leading edge. This is further discussed in $\S 6$.

Turbulence effects for the torsional motion are presented for the lockin and divergence regions in Figs.10 and 11. For the lock-in response (i.e. 
reduced velocity within 4-6), the turbulence effects have a similar trend to that of the heaving motion, suggesting that a similar mechanism influences the structural response for the pitching motion. For the torsional divergence (i.e. reduced velocity beyond 10), many of the characteristics of the VIV response are evident, such as the large magnitude of the response. Regardless of the turbulence parameters considered in the paper, the amplitude keeps increasing beyond the reduced velocity 12. Nevertheless, Figs.10 and 11 also show that the gradient in the divergence region significantly decreases monotonously with the increase of the turbulence intensities and the decrease of the integral length scales.

\section{Spanwise correlation of pressure across the bridge}

Dependency of the spanwise correlations on the magnitude of structure oscillation, and freestream turbulence intensity and integral length scales is the focus of this section. Probes were placed at equal distance along the span at the centre-line of the side face for sampling instantaneous surface pressure. The corresponding spanwise correlation for static pressure was defined as

$$
R_{p}^{z}(\Delta z)=\frac{\overline{p(z, t) \cdot p(z+\Delta z, t)}}{\sqrt{\overline{\overline{p(z, t)^{2}}} \sqrt{\overline{p(z+\Delta z, t)^{2}}}}} .
$$

The correlation coefficient $R_{p}^{z}(\Delta z)$ is plotted against spanwise separation $\Delta z / D$ in Fig. 12 for the heaving case in smooth and turbulent flows. The smooth flow cases are plotted for reduced velocity within (i.e. $U_{r}=8.43$ ) and outside (i.e. $\left.U_{r}=5.93 \& 11.93\right)$ the lock-in region. The pressure correlation for smooth flow at lock-in shows a nearly constant large value $(\sim 0.9)$ 
for all of the separations $\Delta z / D$. This is a similar to that of Bearman and Obasaju (1982) $(\mathrm{B} / \mathrm{D}=1)$ and Ricciardelli $(2010)(B / D=5)$ under a forced vibration. Outside the lock-in, e.g. at $U_{r}=5.93$ and 11.93, the spanwise correlation coefficient of the surface pressure decreases significantly in accordance to the largely reduced vibrational amplitude (Fig. 2). These suggest that the spanwise correlation is dominated by the motion magnitude of the structure.

Similar trend was also observed for the torsional responses. It is not presented in this paper due to limited space.

The turbulence effects on the spanwise correlation of surface pressure within the lock-in region (i.e. $U_{r}=8.4$ ) are also presented in Fig.12. It has already been reported in the literature (e.g. Haan (2000)) that the presence of freestream turbulence intensity diminishes the spanwise correlation for both static, and forced vibration. Fig.12 clearly confirms this observation. The spanwise correlation coefficient of case 'Turbulent -double base $I_{1}, I_{2}, I_{3}$ ' is nearly half of that of case 'Turbulent -base settings'; whereas for case 'Turbulent -halved base $I_{1}, I_{2}, I_{3}$ ' the spanwise correlation coefficient is significantly increased. These are consistent with Fig.8 assuming that large spanwise correlation of surface pressure leads to large structure deformation.

As discussed in $§ 1.1$, in a freestream turbulent flow the structure response can be magnified compared to that in smooth flow (So et al. (2008)). Wu and Kareem (2012) speculated that freestream turbulence can stabilize or destabilize the response. It may help to understand this by looking into the relation between the integral length scales of turbulence and the spanwise correlation of surface pressure fluctuations. Fig.12 shows that the spanwise 
correlation of surface pressure fluctuations increases significantly with the doubled integral length scales. This is consistent with the increase of amplitude of the structure response (Fig.7). However, it is to be noted that the increase of the response is much smaller compare to the increase of the spanwise correlation of the surface pressure fluctuations. Fig.12 also shows that the spanwise correlation of surface pressure fluctuations decreases significantly with the halved integral length scales. Again, the decrease of the structure response with the halved integral length scales is much smaller compared to the decrease of the spanwise correlation of surface pressure.

Overall, it is evident that there is a relation between integral length scales of the freestream turbulence and the spanwise correlation of surface pressure, and subsequently the structural response. Based on the analysis for the formation of the MIV in $\S 4.1$, it can be deduced that the spanwise correlation of surface pressure is the result of MIV convecting along the cylinder which is affected by the freestream turbulence.

To demonstrate this, Fig.13 presents iso-surfaces of vorticity around the cylinder at lock-in under smooth and turbulent flows. For the smooth flow, clearly a two-dimensional MIV is formed across the span resulting in a large correlation in pressure as it convects along the cylinder, whereas for the turbulent case, the MIV breaks down due to the incoming turbulence, diminishing the spanwise correlation of the surface pressure and subsequently the structural response.

For a deeper insight into the vortex formation over the cylinders surface, Fig.14 shows the oilfilm plots of vorticity magnitude over the upper surface of the cylinder for the heaving motion. The diagrams correspond to the 
cylinder at its peak (positive) position of one cycle in the lock-in regime (reduced velocity $U_{r}=8.4$ ), with the oscillation amplitude $y / D=0.05$ (Fig.3). Fig.14(a) shows the flow patterns for the cylinder situated in a smooth flow. It can be seen in this figure that the MIV formed at the leading edge (e.g. vortex 'A1 in Fig.3(ii)) has a two-dimensional structure along the span; this can also be seen in Fig.13(A). At the same time, towards the trailing edge $(x>2 B / 3)$, another region can be identified where the vortices formed from the previous cycle convect into the wake. In this region, some correlation along the span is evident. However, it should be noted that this is not as two-dimensional as the vortex at the leading edge.

In Fig.14(b), it can be seen that the disturbances induced by the freestream turbulence breaks down the MIV 2D structure across the span, whereas the MIV's length along the cylinders chord is slightly increased compared to Fig.14(b). The re-attachment zone seems more irregular compared to Fig.14(b). The region towards the trailing edge also appears to have more three-dimensional characteristic than the equivalent region in the smooth flow case. In summary, for both the smooth incoming flow and the freestream turbulence, the MIVs identified along the cylinders chord is a recurring phenomenon, albeit distorted by the perturbations of the incoming flow.

\section{Conclusions}

The numerical analysis of a rectangular cylinder undergoing Vortex-Induced Vibrations (VIV) under smooth and free turbulent flows is presented. Overall, the conclusions from this research can be summarised:

- An appealing aspect of this work is the analysis of free vibrations, as 
opposed to forced forced vibrations. The latter are more commonly reported in the literature, particularly for experimental analysis.

- The numerical data of 1DOF heaving and torsional motions were compared with the equivalent wind tunnel experimental data.

- With respect to the freestream turbulent flows, the increase of turbulent intensity (less than 12\%, and with the integral length scales in the same order of magnitude of the bridge width $B$ ) has shown to significantly diminish the amplitude of oscillation. This was observed for both resonant responses (VIV) and diverging responses (torsional flutter). It is to be noted that for a stationary cylinder the turbulence intensity of the free turbulent flow enhances the peak loading (e.g. Melbourne (1980), Daniels et al. (2013)).

- The increase of turbulent integral length scales (in the same order of magnitude of the bridge width $B$ ) of the freestream flow moderately enhance the amplitude of oscillation of both heaving and pitching motions. The enhanced amplitudes are less than those in smooth flows. It might be extrapolated from this study that a further increase of the integral length scales will enhance the amplitudes of oscillation to exceed those in smooth flows. To confirm this, it is extremely challenging using wind tunnel experiments and is very computationally expensive using LES.

- The study of spanwise correlation of surface pressure confirms that the increase of the integral length scales of the free turbulence enhances 
the spanwise correlation, and subsequently enhance the the amplitude of oscillation within the lock-in regime.

Again, investigations into greater length scales, to simulate very large turbulence eddies observed in the atmospheric boundary layer are worth further study.

Acknowledgements: This project is supported by an EPSRC Case studentship and partly sponsored by Ove Arup and Partners Ltd. We thank Drs Steven Downie and Ngai Yeung, and Mr Andrew Allsop of Ove Arup and Partners Ltd for their support throughout. The computations were performed on the Iridis4, University of Southampton. SJD is also grateful to Dr Ender Ozkan of RWDI for his useful comments.

\section{References}

G. Bartoli and M. Righi. Flutter mechanism for rectangular prisms in smooth and turbulent flow. Journal of Wind Engineering and Industrial Aerodynamics, 94:275-291, 2006.

P. W. Bearman and E. D. Obasaju. An experimental study of pressure fluctuations on fixed and oscillating squaresection cylinders. Journal of Fluid Mechanics, 119:297-321, 1982.

H.M. Blackburn and W.H. Melbourne. Sectional lift forces for an oscillating circular cylinder in smooth and turbulent flows. Journal of Fluids and Structures, 11:413-431, 1997.

L. Bruno and D. Fransos. Probabilistic evaluation of the aerodynamic prop- 
erties of a bridge deck. Journal of Wind Engineering and Industrial Aerodynamics, 99:718-728, 2011.

L. Bruno, D. Fransos, N. Coste, and A. Bosco. 3d flow around a rectangular cylinder: A computational study. Journal of Wind Engineering and Industrial Aerodynamics, 98:263-276, 2010.

L. Bruno, N. Coste, and D. Fransos. Simulated flow around a rectangular 5:1 cylinder: Spanwise discretisation effects and emerging flow features. Journal of Wind Engineering and Industrial Aerodynamics, 106-106:203$215,2012$.

I. P. Castro, H. Cheng, and R. Reynolds. Turbulence over urban-type roughness: Deductions from wind-tunnel measurements. Boundary-Layer Meteorology, 118(1):109-131, 2006.

S.J. Daniels, I.P. Castro, and Z.T. Xie. Peak loading and surface pressure fluctuations of a tall model building. Journal of Wind Engineering and Industrial Aerodynamics, 120:19-28, 2013.

ESDU-85020. Characteristics of atmosphere turbulence near the ground, part II: single point data for strong winds (neutral atmosphere). Technical Report 85020, Engineering Sciences Data Unit (ESDU), 2001.

D. Fransos and L. Bruno. Edge degree-of-sharpness and free-stream turbulence scale effects on the aerodynamics of a bridge deck. Journal of Wind Engineering and Industrial Aerodynamics, 98:661-671, 2010.

A. Fujiwara, H. Kataoka, and M. Ito. Numerical simulation of flow field 
around an oscillating bridge using finite difference method. Journal of Wind Engineering and Industrial Aerodynamics, 46-47:567-575, 1993.

F.L. Haan. The effects of turbulence on the aerodynamics of long-span bridges. PhD thesis, University of Notre Dame, Department of Aerospace and Mechanical Engineering, Indiana, 2000.

M. Inagaki, T. Kondoh, and Y. Nagano. A mixed time-scale sgs model with fixed model-parameters for practical les. Journal of Fluids Engineering, 127, 1-13 2005.

R. I. Issa. Solution of the implicitly discretised fluid flow equations by operator-splitting. Journal of Comptational Physics, 62:40-65, 1985.

H. Jasak. Error analysis and estimation for the finite volume method with application to fluid flows. PhD thesis, Thermofluids section, Department of Mechanical Engineering, Imperical College of Science, Technology and Medicine, 1996.

J. Jasak and Z. Tuković. Automatic mesh motion for the unstructured finite volume method, 2004. ISSN: 1333-1124.

M. Kawatani, N. Toda, M. Sato, and H. Kobayashi. Vortex-induced torsional oscillations of bridge girders with basic sections in turbulent flows. Journal of Wind Engineering and Industrial Aerodynamics, 83:327-336, 1999.

Y. Kim and Z.T. Xie. Modelling the effect of freestream turbulence on dynamic stall of wind turbine blades. Computers and Fluids, DOI: 10.1016/j.compfluid.2016.02.004, 2016. 
Y. Kim, Z.T. Xie, and I.P. Castro. A forward stepwise method of inflow generation for les. Sixth International Conference on Fluid Mechanics, US, American Institute of Physics, 137-139., 2011.

Y. Kim, I.P. Castro, and Z.T. Xie. Divergence-free turbulence inflow conditions for large-eddy simulations with incompressible flow solvers. Computers and Fluids, 84:56-68, 2013.

S. Komatsu and H. Kobayashi. Vortex-induced oscillation of bluff cylinders. Journal of Wind Engineering and Industrial Aerodynamics, 6(3-4):335$362,1980$.

S. Lee, J.S. Lee, and J.D. Kim. Prediction of vortex-induced wind loading on long-span bridges. Journal of Wind Engineering and Industrial Aerodynamics, 46-47:587-594, 1997.

Q.S. Li and W.H. Melbourne. An experimental investigation of the effects of free-stream turbulence on streamwise surface pressures in separated and reattaching flows. Journal of Wind Engineering and Industrial Aerodynamics, 54/55:313-323, 1995.

Q.S. Li and W.H. Melbourne. The effect of large-scale turbulence on pressure fluctuations in separated and reattaching flows. Journal of Wind Engineering and Industrial Aerodynamics, 83:159-169, 1999.

Y-Y. Lin, C-M. Cheng, J-C. Wu, T-L. Lan, and K-T. Wu. Effects of deck shap and oncoming turbulence on bridge aerodynamics. Tamkang Journal of Science and Engineering, 8(1):43-56, 2005. 
T.S. Lund, X. Wu, and K.D. Squires. Generation of turbulent inflow data for spatially-developing boundary layer simulations. Journal of Computational Physics, 140:233-258, 1998.

C. Mannini, A.M. Marra, and G. Bartoli. Viv-galloping instabilty of rectangular cylinders: Review and new experiments. Journal of wind engineering and industrial aerodynamics, 132:109-124, 2014.

A.M. Marra, C. Mannini, and G. Bartoli. Van der pol-type equation for modeling vortex-induced oscillations of bridge decks. Journal of Wind Engineering and Industrial Aerodynamics, 99:776-785, 2011.

A.M. Marra, C. Mannini, and G. Bartoli. Measurements and improved model of vortex-induced vibration for an elongated rectangular cylinder. Journal of Wind Engineering and Industrial Aerodynamics, 147:358-367, 2015.

M. Matsumoto. Aerodynamic damping of prisms. Journal of Wind Engineering and Industrial Aerodynamics, 59:159-197, 1996.

M. Matsumoto. Vortex effect on torsional flutter. In The seventh AsiaPacific conference on wind engineering, Taipei, Taiwan, November 2009. APCWE-VII.

M. Matsumoto, N. Shiraishi, S. Stoyanoff, and T. Yagi. Mechanism of, and turbulence effect on vortex-induced oscillations for bridge box girders. Journal of Wind Engineering and Industrial Aerodynamics, 49(1-3): 467-476, 1993.

M. Matsumoto, H. Shirato, T. Yagi, R. Shijo, A. Eguchi, and H. Tamaki. 
Effects of aerodynamic interferences between heaving and torsional vibration of bridge decks: the case of the tacoma narrows bridge. Journal of Wind Engineering and Industrial Aerodynamics, 91:1547-1557, 2003.

M. Matsumoto, T. Yagi, H. Tamaki, and T. Tsubota. Vortex-induced vibration and its effect on torsional flutter instability in the case of $\mathrm{b} / \mathrm{d}=4$ rectangular cylinder. Journal of Wind Engineering and Industrial Aerodynamics, 96:971-983, 2008.

W. H. Melbourne. Comparison of measurements on the caarc standard tall building model in simulated model wind flows. Journal of Wind Engineering and Industrial Aerodynamics, 6(1-2):73-88, 1980.

A. Placzek, R. Sigrist, and A. Hamdouni. Numerical simulation of an oscillating cylinder in cross-flow at low reynolds number: forced and free oscillations. Computers and Fluids, 38:80-100, 2008.

F. Ricciardelli. Effects of vibration regime on the spanwise correlation of the aerodynamics forces on a 5:1 rectangular cylinder. Journal of Wind Engineering and Industrial Aerodynamics, 98:215-225, 2010.

T. Sarpkaya. A critical review of the intrinsic nature of vortex-induced vibrations. Journal of Fluids and Structures, 19:389-447, 2004.

M.W. Sarwar and T. Ishihara. Numerical study on suppression of vortexinduced vibrations of box girder bridge section by aerodynamic countermeasures. Journal of Wind Engineering and Industrial Aerodynamics, 98: $701-711,2010$. 
M.W. Sarwar, T. Ishihara, K. Shimada, Y. Tamasaki, and T. Ikeda. Prediction of aerodynamic characteristics of a box girder bridge section using the les turbulence model. Journal of Wind Engineering and Industrial Aerodynamics, 96:1895-1911, 2008.

K. Shimada and T. Ishihara. Application of a modified $k-\epsilon$ model to the prediction of aerodynamic characteristics of recangular cross-section cylinders. Jounral of Fluids and Structures, 16:465-485, 2002.

K. Shimada and T. Ishihara. Predictability of unsteady two-dimensional k-e model on the aerodynamic instabilities of some rectangular prisms. Journal of Fluids and Structures, 28:20-39, 2012.

N. Shiraishi and M. Matsumoto. On classification of vortex-induced oscillation and its application for bridge structures. Journal of Wind Engineering and industrial Aerodynamics, 14(1-3):419-430, 1983.

R.M.C. So, X.Q. Wang, W-C. Xie, and J. Zhu. Free-stream turbulence effects on vortex-induced vibration and flow-induced force of an elastic cylinder. Journal of Fluids and Structures, 24:481-495, 2008.

T. Tamura, K. Nozawa, and K. Kondo. Aij guide for numerical prediction of wind loads on buildings. Journal of Wind Engineering and Industrial Aerodynamics, 96(10-11):1974-1984, 2008.

C.H.K. Williamson and R. Govardhan. vortex-induced vibrations. Annual review of Fluid Mechanics, 36(1):413-455, 2004.

T. Wu and A. Kareem. An overview of vortex-induced vibration (viv) of 
821 bridge decks. Journal of Frontiers of Structural Civil Engineering, 6(4): $822 \quad 335-347,2012$.

${ }_{823}$ Z.T. Xie and I.P. Castro. Efficient generation of inflow conditions for large 824 eddy simulation of street-scale flows. Flow, Turbulence and Combustion, $825 \quad 81(3): 449-470,2008$. 


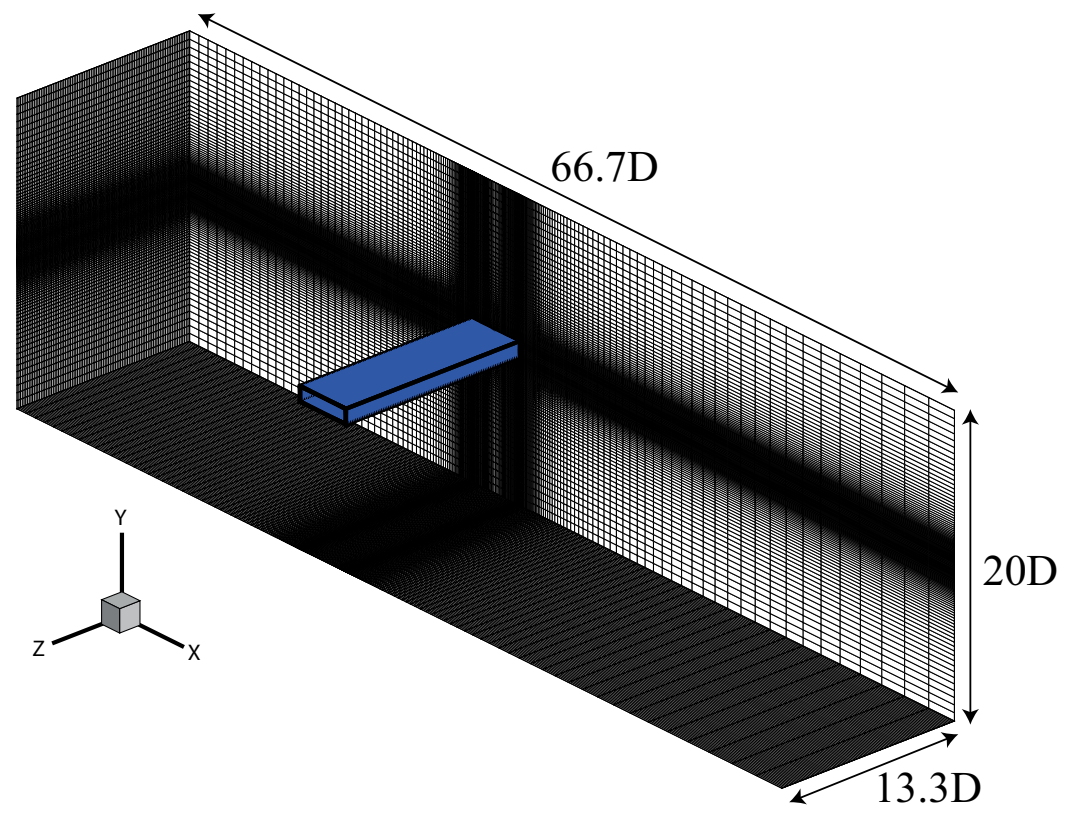

Figure 1: Overall grid distribution for the 3-dimensional case $\left(y_{1}^{+}<5\right)$, with dimensions, and coordinates $\mathrm{x}, \mathrm{y}, \mathrm{z}$ corresponding to streamwise, vertical and spanwise respectively. The origin of the reference system is placed at the left bottom corner of the inlet plane when looking downstream. 


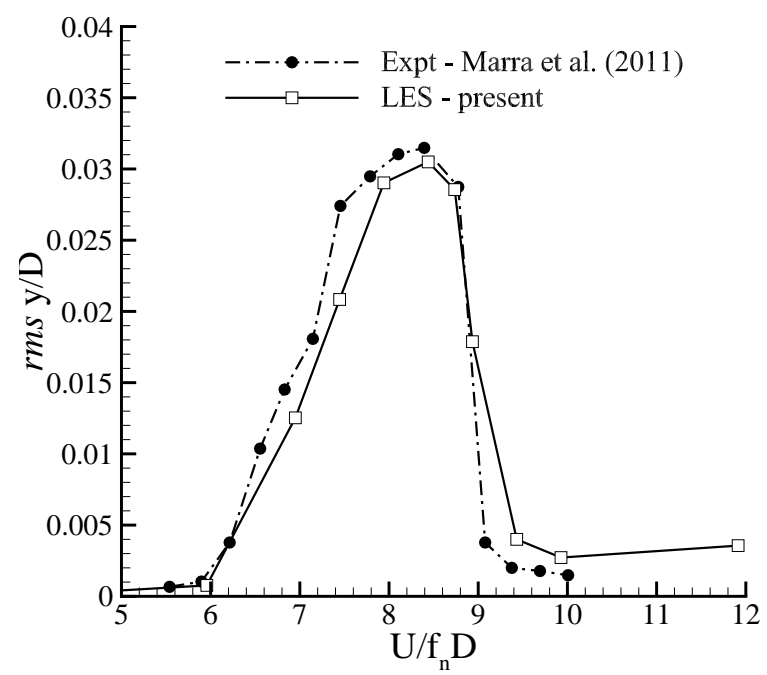

Figure 2: Root-mean-squared (r.m.s) non-dimensional deflection versus reduced velocity $U / f_{n} D$ in smooth flow $\left(f_{n}=\right.$ vertical natural frequency $)$. 

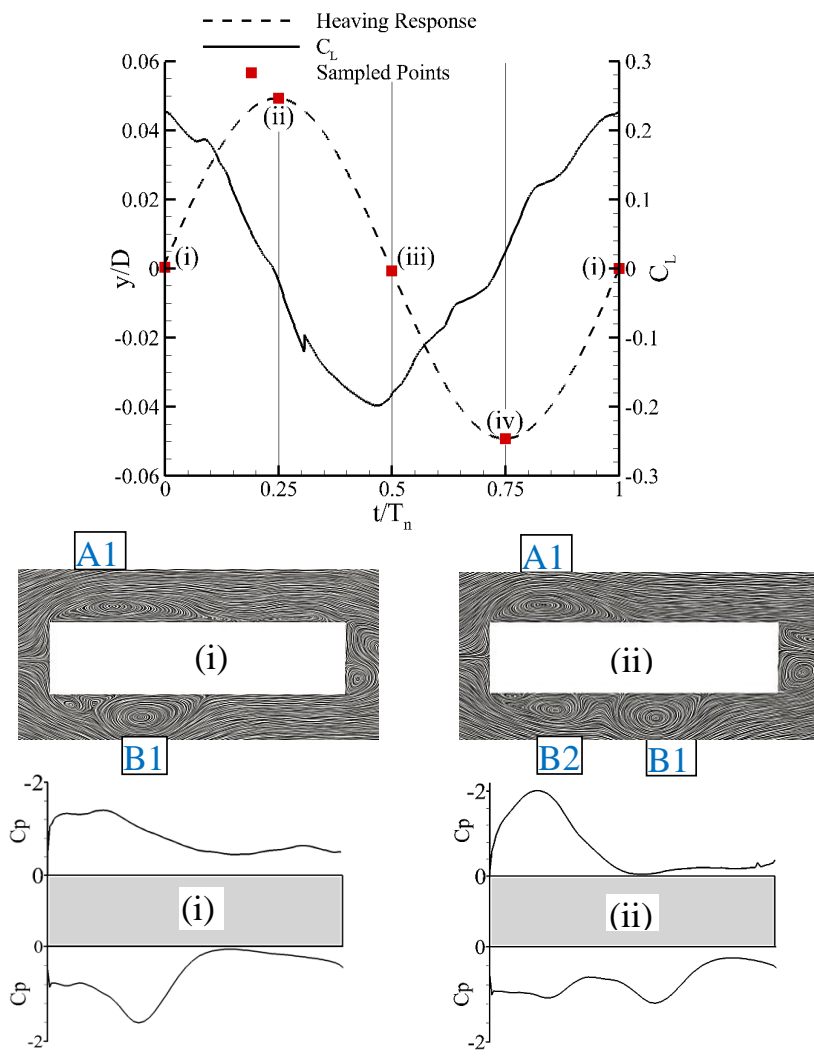

\section{A1}
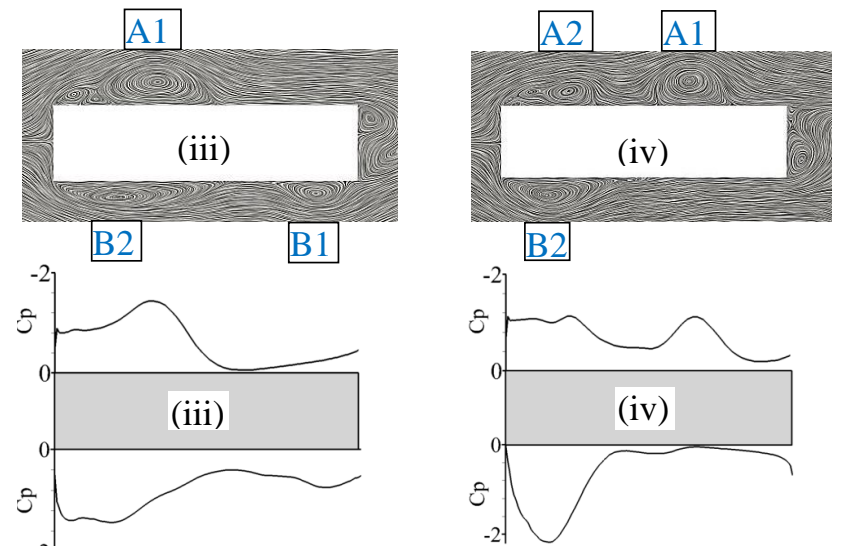

Figure 3: Phase-averaged streamlines and pressure distribution over upper and lower surface of the cylinder during one cycle at lock-in for 1DOF heaving motion. $U / f_{n} D=8.4$. Top: phase-averaged lift and deflection time series over one cycle. $C_{L}=L / 0.5 \rho U^{2} B$ and $C_{p}=\left(p-p_{\infty}\right) / 0.5 \rho_{f} U^{2}$. Figs. (i)-(iv) respectively correspond to phase angles $0^{\circ}, 90^{\circ}, 180^{\circ}$ and $270^{\circ}$. 


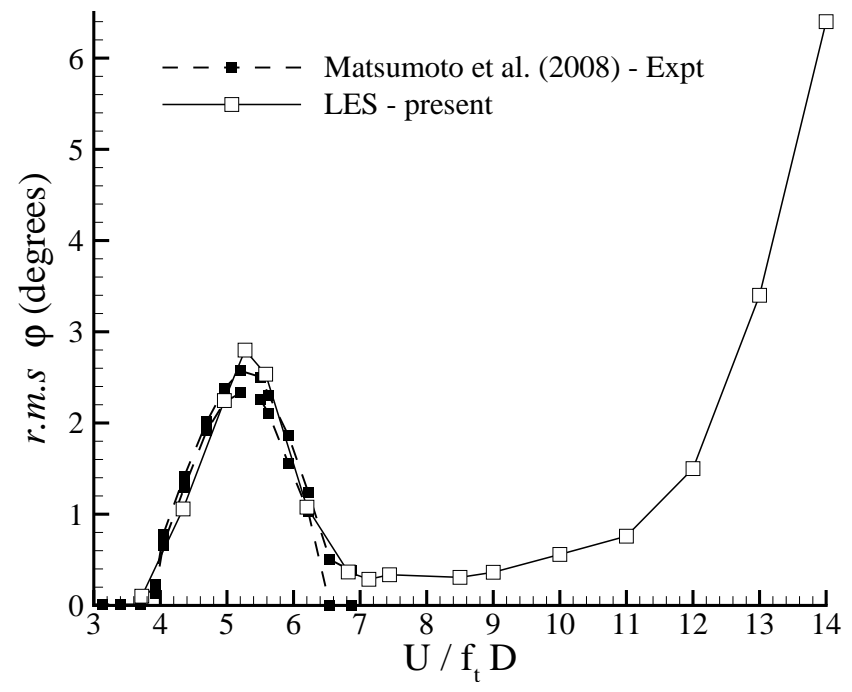

Figure 4: Root-mean-squared (r.m.s) pitching angle versus reduced velocity $\left(U / f_{t} D\left(f_{t}=\right.\right.$ torsional natural frequency). The rotational axis is the mid-chord. 

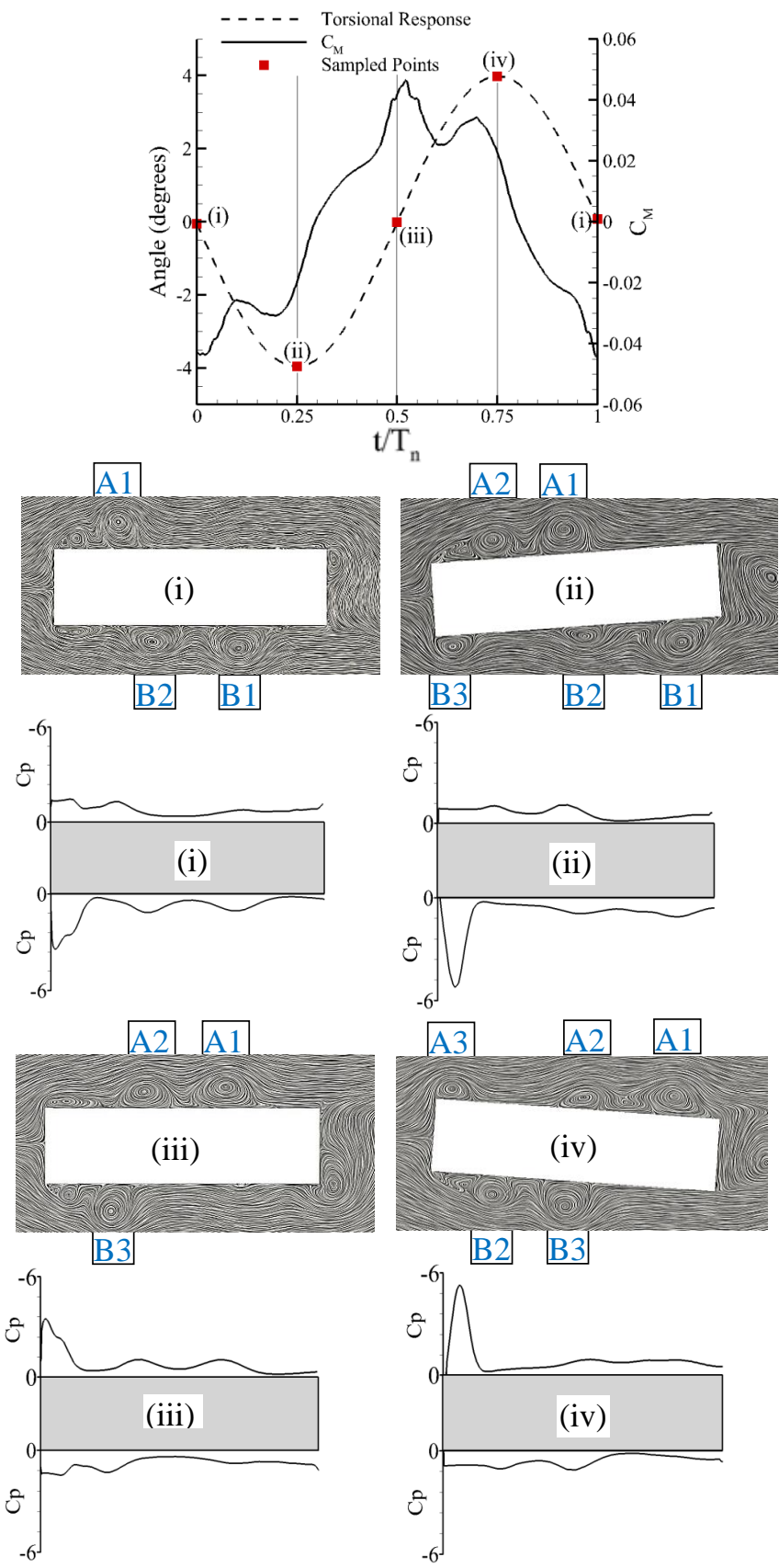

Figure 5: Phase-averaged streamlines and pressure distribution over upper and lower surfaces of the cylinder during one cycle at lock-in for 1DOF pitching with rotational around the mid-chord. Reduced velocity $U / f_{t} D=4.9$. Top: phase-averaged pitching moment and angle phase-averaged time series over one cycle. $C_{M}=M / 0.5 \rho U^{2} B^{2}$. (i)(iv) respectively correspond to phase angles $0^{\circ}, 90^{\circ}, 180^{\circ}$ and $270^{\circ}$. 


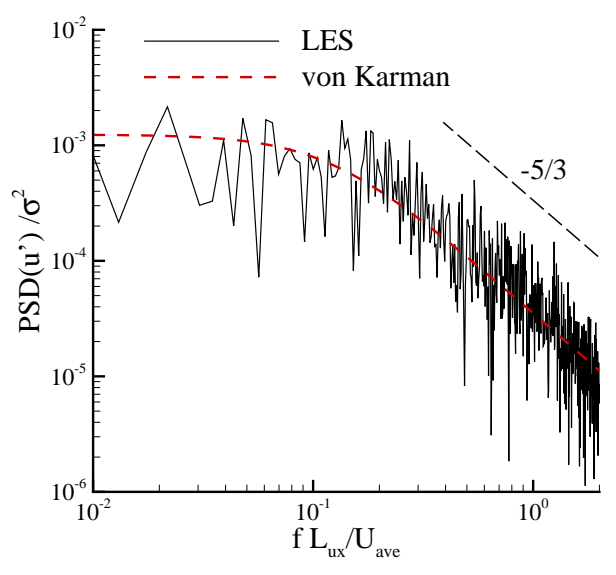

(a)

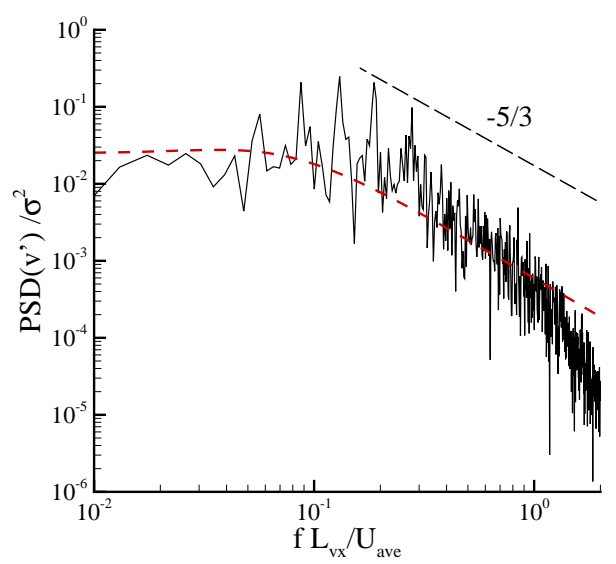

(b)

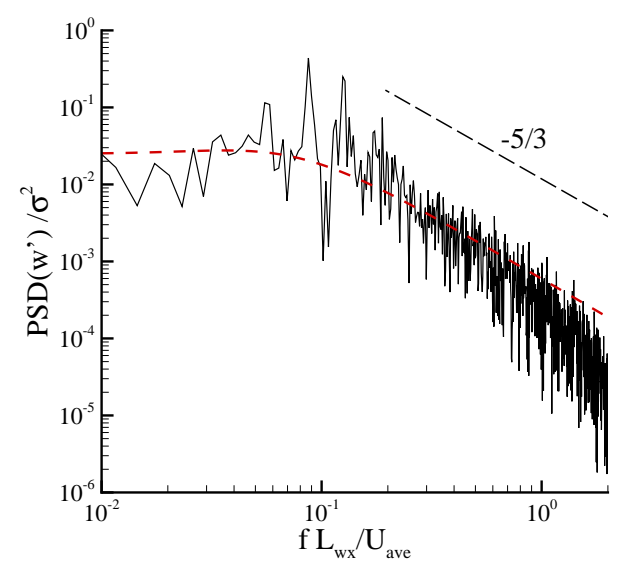

(c)

Figure 6: Power spectral density of velocity fluctuations at $x=3 B, \mathrm{y}=2.5 \mathrm{~B}$ on the central plane. (a) $u^{\prime}$, (b) $v^{\prime}$, (c) $w^{\prime}$. 


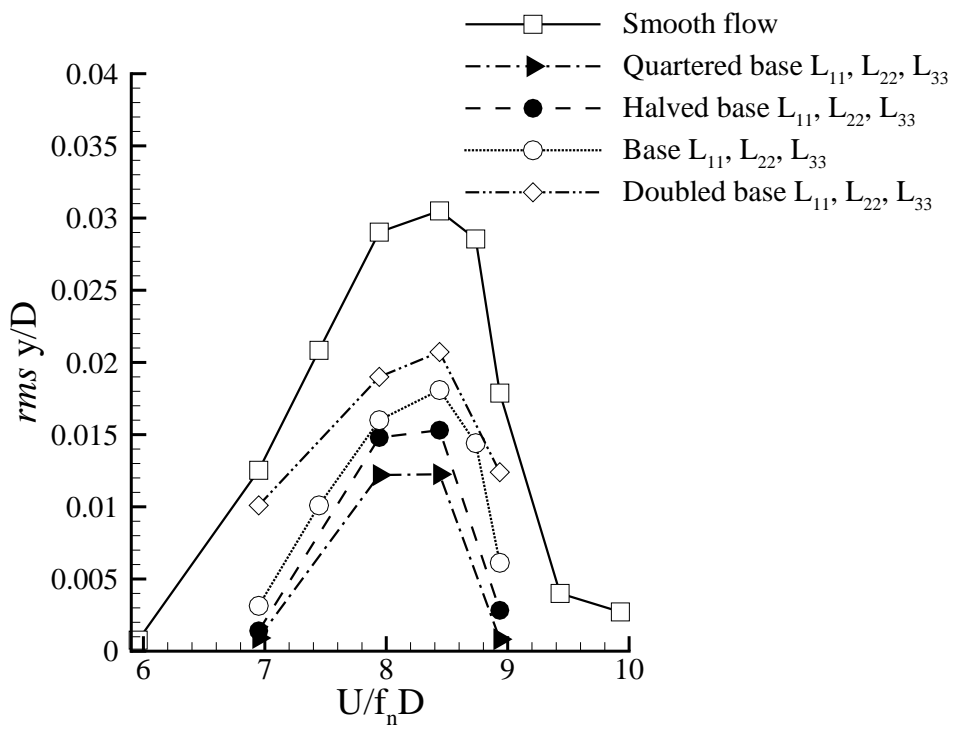

Figure 7: Root-mean-squared (r.m.s) non-dimensional deflection versus reduced velocity $U / f_{n} D$ under a turbulent flow with various integral length scales.

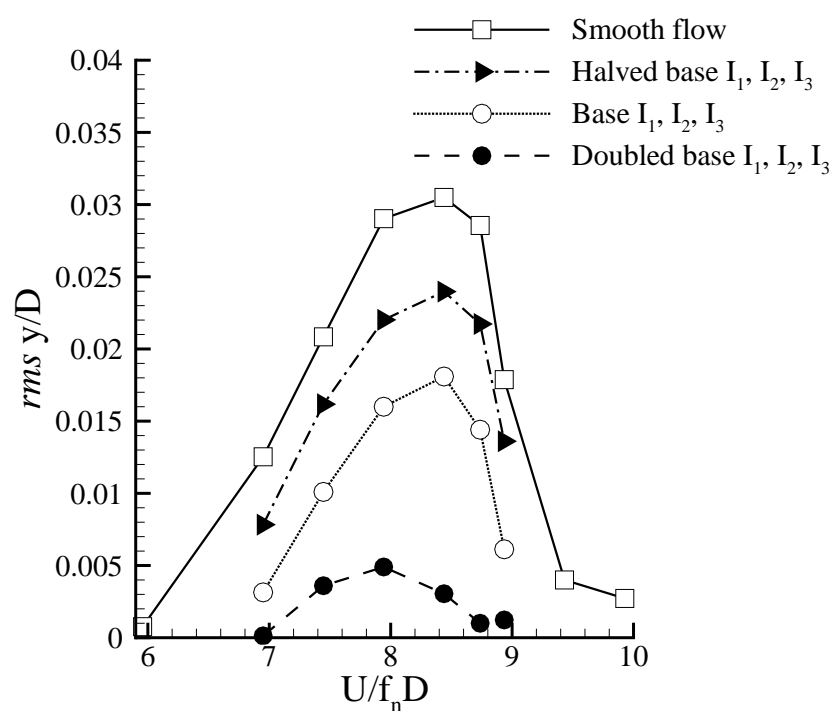

Figure 8: Root-mean-squared (r.m.s) non-dimensional deflection versus reduced velocity $U / f_{n} D$ under a turbulent flow with various turbulence intensities. 


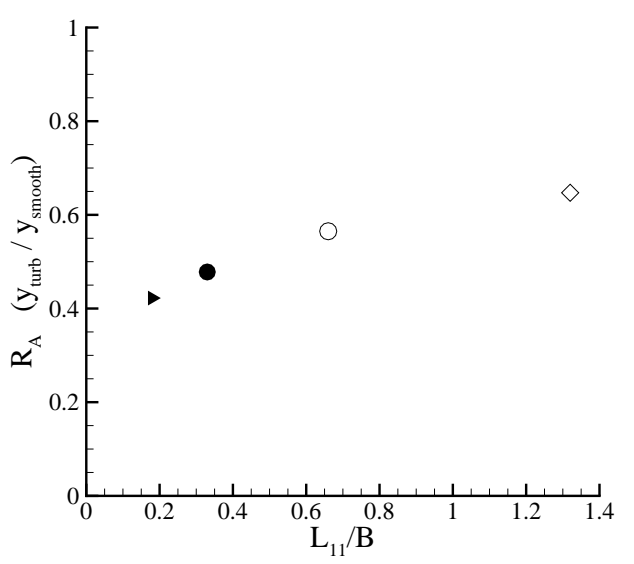

(a)

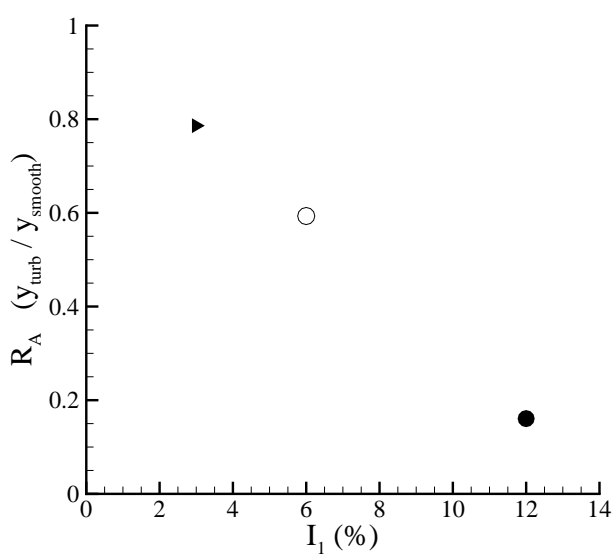

(b)

Figure 9: (a) Amplitude ratio versus turbulence length scale $L_{11}$; the legend for the symbols as Fig. 7. (b) Amplitude ratio versus turbulence intensity $I_{1}$; the legend for the symbols as Fig. 8. $U / f_{n} D=8.4$.

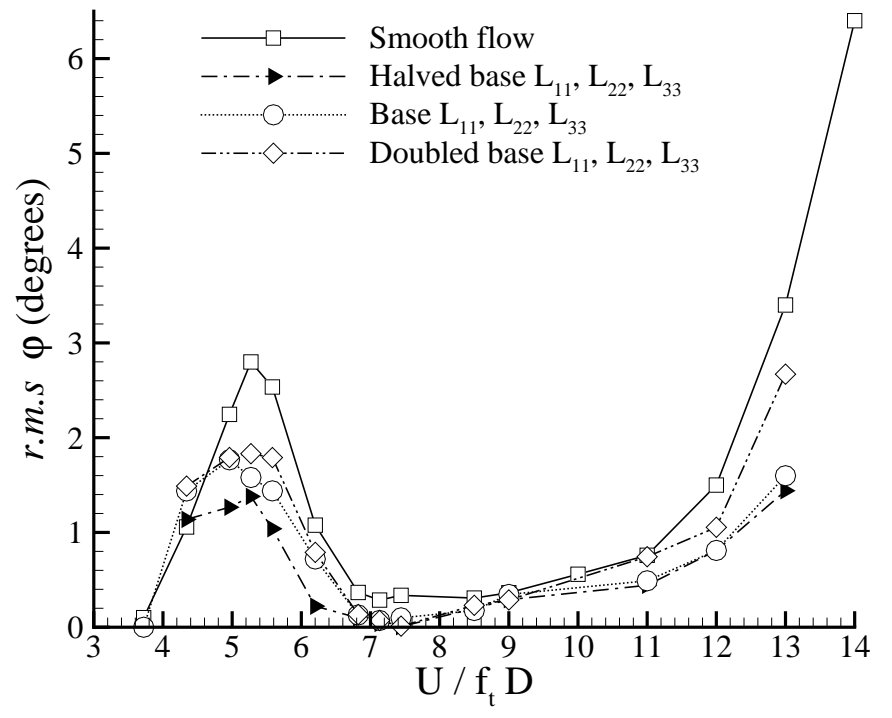

Figure 10: Root-mean-squared (r.m.s) pitching angle versus reduced velocity under a turbulent flow with various integral length scales. 


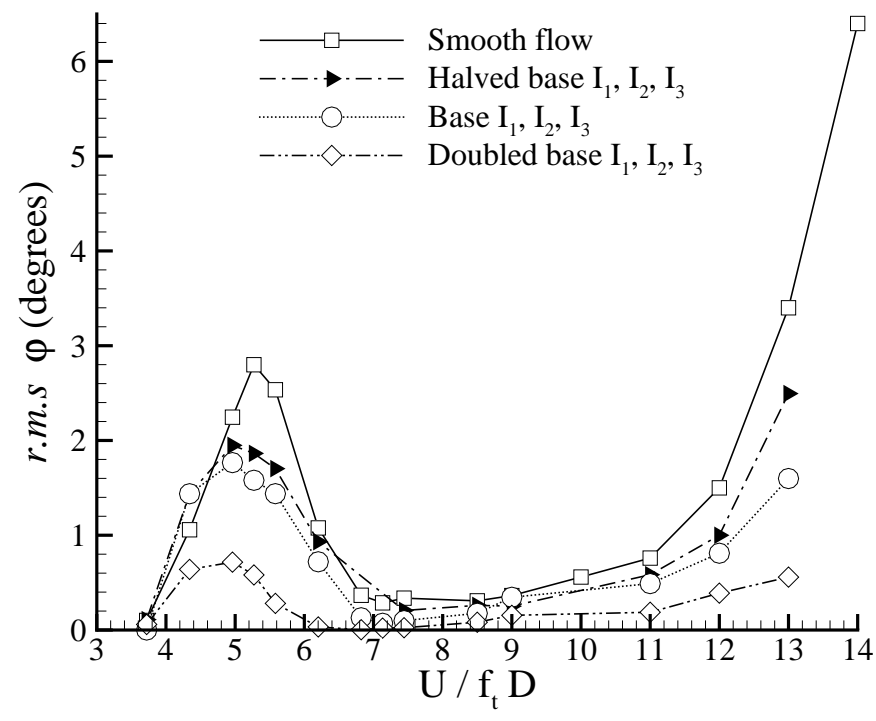

Figure 11: Root-mean-squared (r.m.s) pitching angle versus reduced velocity under a turbulent flow with various turbulence intensities. 


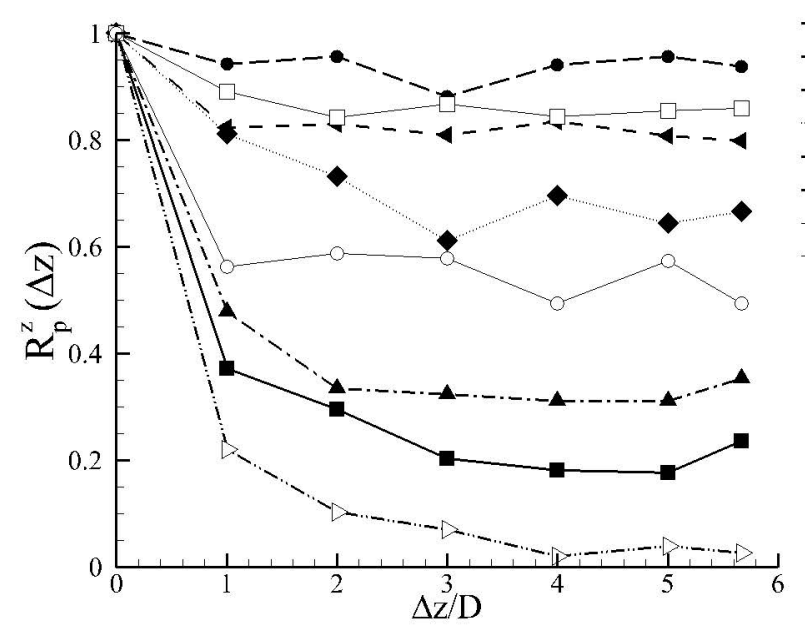

$\longrightarrow$ Smooth $-\mathrm{U}_{\mathrm{r}}=5.93$

- - - Smooth - $\mathrm{U}_{\mathrm{r}}=8.43$ (lock-in)

$-\cdots-\cdots-\cdots-\quad$ Smooth $-\mathrm{U}_{\mathrm{r}}=11.93$

………..... Turbulent - base settings

-.-A-_-A Turbulent - doubled base $\mathrm{I}_{1}, \mathrm{I}_{2}, \mathrm{I}_{3}$

- - 4 - - Turbulent - doubled base $\mathrm{L}_{11}, \mathrm{~L}_{22}, \mathrm{~L}_{33}$

$\square \quad$ Turbulent - halved base $\mathrm{I}_{1}, \mathrm{I}_{2}, \mathrm{I}_{3}$

$\multimap$ Turbulent - halved base $\mathrm{L}_{11}, \mathrm{~L}_{22}, \mathrm{~L}_{33}$

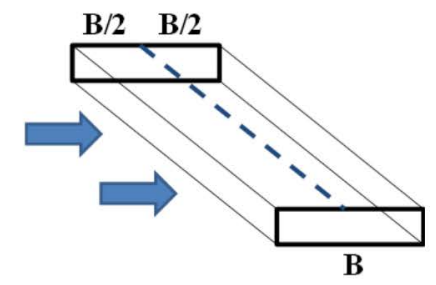

Figure 12: Spanwise correlation of pressure on the centre of the side face for both static and heaving cases under smooth and turbulent flows. $U_{r}=U / f_{n} D$ is reduced velocity. All turbulent cases are for $U_{r}=8.4$ (lock-in). 

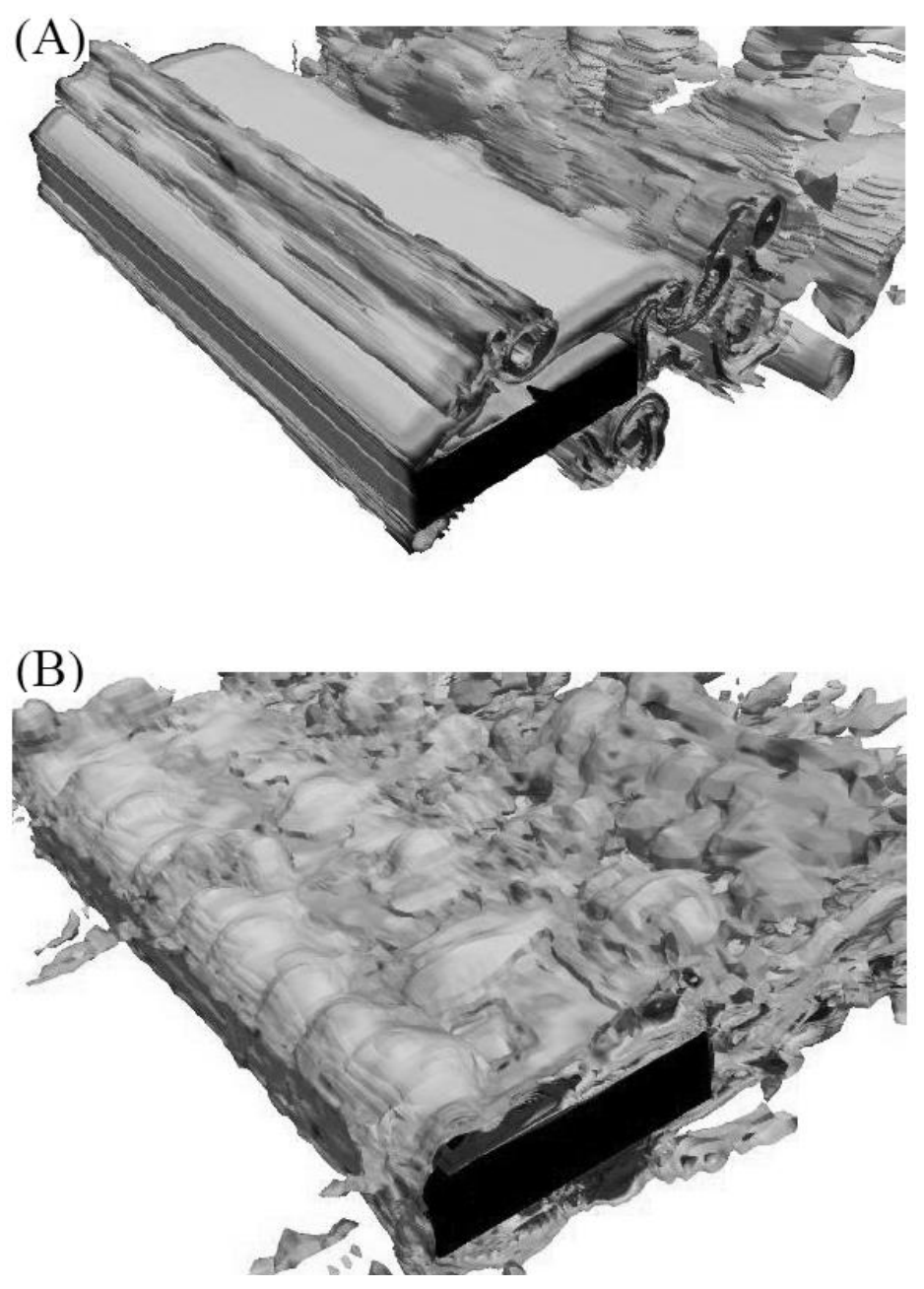

Figure 13: Instantaneous iso-surfaces of the vorticity magnitude $(-100,100)\left(s^{-1}\right)$. Dynamic cases at lock-in (A) under smooth, (B) under turbulent flow. 


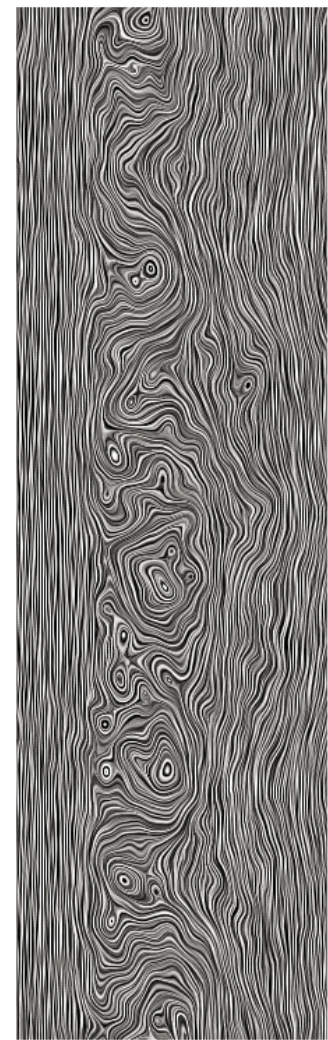

(a) Smooth flow.

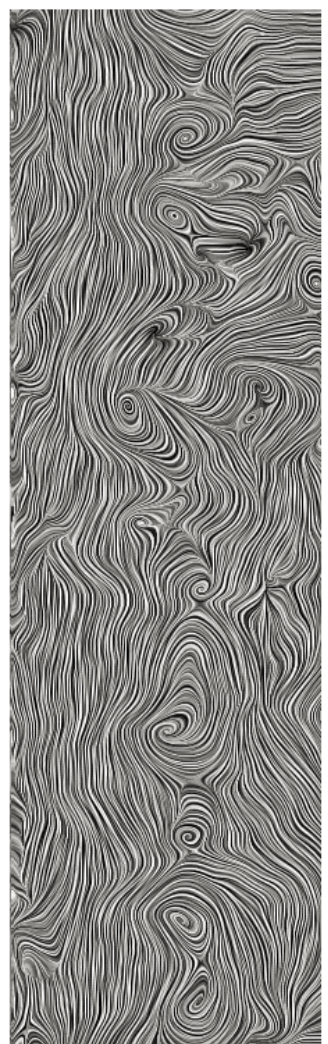

(b) Turbulent flow with base settings.

Figure 14: Oilfilm plots of vorticity magnitude over the cylinders surface for the heaving motion in the lock-in regime. Flow is from the left to the right. 